\title{
Correction to: LI-RADS technical requirements for CT, MRI, and contrast-enhanced ultrasound
}

\author{
Avinash R. Kambadakone $\odot,{ }^{1}$ Alice Fung, ${ }^{2}$ Rajan T. Gupta, ${ }^{3}$ Thomas A. Hope, ${ }^{4}$ \\ Kathryn J. Fowler, ${ }^{5}$ Andrej Lyshchik, ${ }^{6}$ Karthik Ganesan, ${ }^{7}$ Vahid Yaghmai, ${ }^{8}$ \\ Alexander R. Guimaraes, ${ }^{2}$ Dushyant V. Sahani, ${ }^{1}$ Frank H. Miller ${ }^{8}$ \\ ${ }^{1}$ Department of Radiology, Massachusetts General Hospital, Harvard Medical School, 55 Fruit Street, White 270, Boston, MA \\ 02114, USA \\ ${ }^{2}$ Department of Diagnostic Radiology, Oregon Health and Science University, Portland, OR, USA \\ ${ }^{3}$ Department of Radiology, Duke University School of Medicine, Durham, NC, USA \\ ${ }^{4}$ Department of Radiology, University of California San Francisco, San Francisco, CA, USA \\ ${ }^{5}$ Department of Radiology, Washington University School of Medicine, St Louis, MO, USA \\ ${ }^{6}$ Department of Radiology, Thomas Jefferson University Hospital, Philadelphia, PA, USA \\ ${ }^{7}$ Department of Radiology, Sir HN Reliance Foundation Hospital and Research Centre, Mumbai, India \\ ${ }^{8}$ Department of Radiology, Northwestern University Feinberg School of Medicine, Chicago, IL, USA
}

\section{Correction to: Abdom Radiol (2017) DOI 10.1007/s00261-017-1325-y}

The original version of this article unfortunately contained mistakes in the author list. The following author names were listed without middle initial. The correct author names are:

Avinash R. Kambadakone,

Rajan T. Gupta,

Thomas A. Hope,

Kathryn J. Fowler,

Alexander R. Guimaraes,

Dushyant V. Sahani,

Frank H. Miller.

The original article was corrected.

The online version of the original article can be found under doi: 10.1007/s00261-017-1325-y.

Correspondence to: Avinash R. Kambadakone; email: akambadakone@mgh.harvard.edu 